Article

\title{
Mini Nutritional Assessment May Identify a Dual Pattern of Perturbed Plasma Amino Acids in Patients with Alzheimer's Disease: A Window to Metabolic and Physical Rehabilitation?
}

\author{
Roberto Aquilani ${ }^{1}$, Alfredo Costa ${ }^{2,3}$, Roberto Maestri ${ }^{4}$, Matteo Cotta Ramusino ${ }^{2,3}$, \\ Antonia Pierobon ${ }^{5}$, Maurizia Dossena ${ }^{1}$, Sebastiano Bruno Solerte ${ }^{6}$, Anna Maria Condino ${ }^{7}$, \\ Valeria Torlaschi ${ }^{5}$, Paola Bini ${ }^{2,3}$, Mirella Boselli ${ }^{8}$, Mauro Ceroni ${ }^{3,9}$, Daniela Buonocore ${ }^{1}$, \\ Federica Boschi ${ }^{7}$, Mariella Bruni ${ }^{10}$ and Manuela Verri ${ }^{1, *}$ \\ 1 Department of Biology and Biotechnology “Lazzaro Spallanzani”, University of Pavia, 27100 Pavia, Italy; \\ dottore.aquilani@gmail.com (R.A.); maurizia.dossena@unipv.it (M.D.); daniela.buonocore@unipv.it (D.B.) \\ 2 Center of Cognitive and Behavioral Disorders, IRCCS Mondino Foundation, 27100 Pavia, Italy; \\ alfredo.costa@mondino.it (A.C.); matteo.cottaramusino@mondino.it (M.C.R.); paola.bini@mondino.it (P.B.) \\ 3 Department of Brain and Behavior, University of Pavia, 27100 Pavia, Italy; mauro.ceroni@unipv.it \\ 4 Department of Biomedical Engineering of the Montescano Institute, Istituti Clinici Scientifici Maugeri IRCCS, \\ 27040 Montescano (PV), Italy; roberto.maestri@icsmaugeri.it \\ 5 Psychology Unit of the Montescano Institute, Istituti Clinici Scientifici Maugeri IRCCS, \\ 27040 Montescano (PV), Italy; antonia.pierobon@icsmaugeri.it (A.P.); valeria.torlaschi@icsmaugeri.it (V.T.) \\ 6 Department of Internal Medicine, University of Pavia, 27100 Pavia, Italy; bruno.solerte@unipv.it \\ 7 Department of Drug Sciences, University of Pavia, 27100 Pavia, Italy; annamaria.condino@unipv.it (A.M.C.); \\ federica.boschi@unipv.it (F.B.) \\ 8 Neurorehabilitation Unit of the Montescano Institute, Istituti Clinici Scientifici Maugeri IRCCS, \\ 27040 Montescano (PV), Italy; mirella.boselli@icsmaugeri.it \\ 9 General Neurology Unit, IRCCS Mondino Foundation, 27100 Pavia, Italy \\ 10 Medical Laboratory Service of the Pavia Institute, Istituti Clinici Scientifici Maugeri IRCCS, \\ 27100 Pavia, Italy; mariella.bruni@icsmaugeri.it \\ * Correspondence: manuela.verri@unipv.it; Tel.: +39-0382-986-423
}

Received: 3 June 2020; Accepted: 18 June 2020; Published: 21 June 2020 updates

\begin{abstract}
Conflicting results about alterations of plasma amino acid (AA) levels are reported in subjects with Alzheimer's disease (AD). The current study aimed to provide more homogeneous AA profiles and correlations between AAs and cognitive tests. Venous plasma AAs were measured in 54 fasting patients with $\mathrm{AD}$ ( 37 males, 17 females; $74.63 \pm 8.03 \mathrm{yrs} ; 3.2 \pm 1.9$ yrs from symptom onset). Seventeen matched subjects without neurodegenerative symptoms (NNDS) served as a control group (C-NNDS). Patients were tested for short-term verbal memory and attention capacity and stratified for nutritional state (Mini Nutritional Assessment, MNA). Compared to C-NNDS, patients exhibited lower plasma levels of aspartic acid and taurine $(p<0.0001)$ and higher 3-methylhistidine $(p<0.0001)$, which were independent of patients' MNA. In comparison to normonourished $\mathrm{AD}$, the patients at risk of and with malnutrition showed a tendency towards lower ratios of Essential AAs/Total AAs, Branched-chain AAs/Total AAs, and Branched-chain AAs/Essential AAs. Serine and histidine were positively correlated with verbal memory and attention capacity deficits, respectively. Total AAs negatively correlated with attention capacity deficits. Stratifying patients with AD for MNA may identify a dual pattern of altered AAs, one due to AD per se and the other linked to nutritional state. Significant correlations were observed between several AAs and cognitive tests.
\end{abstract}

Keywords: Alzheimer's disease; plasma amino acids; nutritional state; cognitive tests 


\section{Introduction}

Amino acid (AA) levels in the blood and cerebrospinal fluid of patients with Alzheimer's disease (AD) were measured to detect biochemical signature for early diagnosis, given that AAs, in addition to their nutritional characteristics, are essential for brain neurotransmission [1] and receptor signalling pathways [2]. Although alterations in AA metabolism in AD have been reported to be an early marker of neurodegeneration [3,4], studies have shown inconsistent results: some have highlighted higher plasma AA levels in AD subjects compared to normal controls, while others have highlighted lower or unchanged levels $[5,6]$. The levels of plasma AAs were also investigated to understand the overtime changes from healthy conditions to subjective memory complaint, to mild cognitive impairment, to AD [7]. Recently, a study documented that levels of blood branched-chain aminotransferase protein (BCAT), the enzyme that regulates Branched Chain AAs (BCAAs) and glutamate metabolism, and glutamate were higher in $\mathrm{AD}$ in comparison to healthy controls. This helped to increase the reliability of neurocognitive assessment in distinguishing healthy subjects and subjects with AD [8].

Epidemiologic studies have found inverse associations between plasma BCAAs and increased risk of all dementias, AD [9], cognitive decline and cerebral atrophy changes [10].

However, in another study, no differences were found in plasma BCAAs among healthy controls, subjects with mild cognitive impairment and subjects with AD [11].

The basic idea behind the current study was that we may be able to achieve more homogenous, reliable plasma AA profile measures by relating plasma AAs to patients' nutritional and metabolic states. Several factors may influence the levels of circulating AAs in AD including both physiological factors such as protein intake and body proteolysis [12,13] and pathological factors such as inflammation [14], in which oral [15] and intestinal [16] dysbioses may play a role, insulin resistance [17], oxidative stress [18], skeletal muscle mitochondrial dysfunction following $\beta$-amyloid (A $\beta$ ) deposition [19-21], and wasting of lean body mass [22].

The reliability of plasma AAs is of clinical importance because AA alterations may suggest body/muscle wasting. In addition, in the presence of AA alterations, we may suspect a progressive failure of neuronal networks and neurotransmitter systems, which causes both synaptic loss and dysfunction [23]. In fact, several circulating AAs, after crossing the blood-brain barrier (BBB), serve as precursors of neurotransmitters in the brain [1]. The AAs cross the BBB in competition with each other [1].

Therefore, in the current observational, prospective study, we measured plasma AA levels in a cohort of subjects with AD. Then, we related the plasma AA levels to both patients' nutritional state according to a clinical assessment of nutritional state (Mini Nutritional Assessment, MNA) and metabolic state such as insulin resistance. We hypothesised that the AD subjects may be characterised by two diverse perturbed patterns of plasma AA levels, one due to their primary disease (AD) per se and the other linked to the deterioration of their nutritional state. The second hypothesis we formulated was that plasma AAs may be associated with patients' cognitive functions.

\section{Materials and Methods}

\subsection{Population}

A total of 71 individuals -17 control subjects and 54 patients with dementia that was possibly due to $\mathrm{AD}$ (37 males, 17 females; $74.63 \pm 8.03 \mathrm{yrs;} 3.2 \pm 1.9$ yrs from symptom onset, according to information supplied by caregivers) - were included in this study. Patients were consecutive cognitively impaired subjects, who underwent diagnostic workup in the Department of General Neurology at the National Institute of Neurology IRCCS Mondino Foundation, Pavia, Italy, between 2014 and 2018. Etiological diagnosis of dementia was formulated based on in vivo evidence of Alzheimer's pathology in cerebrospinal fluid (CSF): a biomarker profile was considered suggestive if $A \beta_{1-42}<599 \mathrm{pg} / \mathrm{mL}$, tau $>404 \mathrm{pg} / \mathrm{mL}$, p-tau $>56.5 \mathrm{pg} / \mathrm{mL}$, or $\mathrm{A} \beta_{1-42} / \mathrm{tau}<1.6, \mathrm{~A} \beta_{1-42-} / \mathrm{p}$-tau $<11.5[24,25]$. Exclusion criteria were the following: history of significant neurological or psychiatric illnesses or presence of other 
diseases precluding enrolment, visual and auditory acuity that was inadequate for neuropsychological testing, and use of drugs with significant central nervous system anticholinergic activity. All stages of AD were represented, with a marked prevalence for mild and moderate AD [26,27]. At the time of blood sampling and CSF collection, some patients were taking choline supplements but not anticholinesterase drugs. With regard to anticholinesterase drugs, $75.9 \%$ of the patients had stopped taking them at least two weeks before blood and CSF sampling, 18.5\% did not take them because of bradycardia, and 5.6\% had not started taking them yet. Moreover, the recruited AD patients were not on corticosteroid nor hormone treatment.

For control group (C) we selected age-matched subjects without cognitive impairment (no neurodegenerative subjects, NNDS; C-NNDS) who underwent neurological consultation and brain MRI scan for reasons unrelated to cognition (paresthesias, migraine or headache, dizziness, auditory disturbances, and lower limb pain). Subjects suffering from diabetes, heart failure, chronic obstructive pulmonary disease, renal disease or endocrinopathies, or involved in discretional physical activity were excluded because all these factors may be responsible for changes in the plasma AA profile. There were ten females and seven males; mean age was $71.80 \pm 6.30$ yrs; body mass index (BMI) was $25.7 \pm 4.9 \mathrm{~kg} / \mathrm{m}^{2}$ (Table 1). Collection and analysis of blood samples were carried out at the same Neurological Institution as for the AD patients.

The study was conducted in accordance with the Declaration of Helsinki, and the protocol was approved by the Ethics Committee of Ospedale San Raffaele-Istituto di Ricovero e Cura a Carattere Scientifico-Milano, Italy (Project identification code: MAIR2016; ethical approval: 130/INT/2016; date: 8 September 2016). All subjects or their legal representatives gave their informed consent for inclusion before they participated in the study. Participants did not receive financial compensation.

\subsection{Procedures}

\subsubsection{Clinical Evaluations}

Enrolled patients all underwent complete clinical, neurological, and neuropsychological assessment and brain magnetic resonance imaging (MRI).

In addition to the physical examination, routine blood tests, and anthropometric measurements (weight, kg; height, $\mathrm{cm}$; body mass index, BMI, $\mathrm{kg} / \mathrm{m}^{2}$; mid-arm circumference, $\mathrm{cm}$ ), clinical evaluation included an assessment of patients' nutritional state by means of a Mini Nutritional Assessment (MNA) score [28]. A score below 17 denotes a state of malnutrition, a score between 17 and 24 denotes risk of malnutrition, and a score above 24 identifies a normal nutritional state.

\subsubsection{Neuropsychological Assessment}

The formal neuropsychological examination included a Mini Mental State Examination (MMSE: a score $<24$ denotes cognitive impairment) and Attentive Matrices. Thus, Digit Span (Digit Span <3.75) [29], which assesses short-term verbal memory, Trail Making Test [30,31] TMT-A (visuospatial selective attention), and Trail Making Test TMT-B (divided attention) were evaluated. Time needed (in seconds) to complete Part A is used as a measure of psychomotor speed (TMT-A < 93"), and time needed to complete Part B is used as a measure of alternation/flexibility, inhibition/interference control, mental tracking, and attentional set-shifting (TMT-B $\left.<282^{\prime \prime}\right)$.

\subsubsection{AD Biomarker Measurements in CSF}

CSF samples were centrifuged for $10 \mathrm{~min}$ at $1800 \times \mathrm{g}$ at $4{ }^{\circ} \mathrm{C}$ within $3 \mathrm{~h}$ of collection. The samples were then divided into aliquots of $0.5 \mathrm{~mL}$ in polypropylene tubes and stored at $-80{ }^{\circ} \mathrm{C}$.

Measurement of CSF $\beta$-amyloid, tau, and p-tau was performed using standardised ELISA (Fujirebio Europe, Ghent, Belgium). 


\subsubsection{Plasma AA Measurements}

In both patients and C-NNDS, at 8 a.m. after $12 \mathrm{~h}$ of overnight fasting, blood samples were drawn from an antecubital vein and immediately delivered to the laboratory where plasma was obtained from heparinised blood using centrifugation $(800 \times g, 15 \mathrm{~min})$.

The concentration of free amino acids in the plasma was measured using an AminoQuant II amino acid analyser, based on the HP 1090 HPLC system, with fully automated precolumn derivatisation. Both orthopthalaldehyde (OPA) and 9-fluorenyl-methyl-chloroformate (FMOC) reaction chemistries were used, according to the manufacturer's protocol. Measurements were made by injecting $1 \mu \mathrm{L}$ of the derivatised mixture and measuring absorbance simultaneously at 338 and $262 \mathrm{~nm}$ [32]. Plasma concentrations were expressed as $\mu \mathrm{mol} / \mathrm{L}$. The measurements of the plasma amino acids were carried out in triplicate by the same laboratory. The mean of the three measurements was calculated and adopted. The characteristics of the method were based both on precision and standardisation properties (unpublished data): precision, relative standard deviation (RSD) was $1.13 \%$; reliability (bias) was $10.37 \%$; the lower limit of quantitation was $0.18 \mathrm{ng} / \mathrm{mL}$; the limit of detection was $0.6 \mathrm{ng} / \mathrm{mL}$. For the measurements in triplicate, the intra-day variability (RSD) was $3.21 \%$ and the intervariability was $4.77 \%$.

The AAs measured were: (1) Essential AAs (EAAs): leucine, valine, isoleucine, lysine, threonine, phenylalanine, methionine, and tryptophan. The first three EAAs constitute the Branched-chain AAs (BCAAs); (2) non-EAAs: aspartic acid, glutamic acid, histidine, asparagine, serine, glutamine, 3-methyl-histidine, arginine, citrulline, glycine, alanine, taurine, and tyrosine.

As AA levels in venous plasma mainly reflect the metabolism of the tissue/organ where the blood is drawn from, we discussed AAs mainly in relation to skeletal muscle tissue.

\subsubsection{Insulin and HOMA-IR}

At 8 a.m., peripheral venous blood samples were drawn for assay of insulin $(\mu \mathrm{U} / \mathrm{mL})$ levels (commercial kits Cord-CT Radioimmunoassay Kit CIS France, Coat A Count Insulin, D.P.C., Los Angeles, CA, USA). Insulin resistance was calculated by the HOmeostasis Model Assessment (HOMA; normal value $<2.4)[33,34]$.

\subsection{Statistical Analysis}

Descriptive statistics are reported as mean \pm SD for continuous data and as a number (percentage frequency) for discrete variables. The Shapiro-Wilk test, supported by visual inspection, was used to assess the normality of the distribution of continuous variables. Since several variables violated the normality assumption, non-parametric statistics were preferred. Accordingly, between group comparisons were carried out by the Kruskal-Wallis test. When three or more groups were compared, if a global significant difference was observed, post-hoc paired comparisons were carried out (Dunn-Sidak test).

The association between neurocognitive tests and plasma AAs, between plasma AAs and CSF concentrations of AD biomarkers, between neurocognitive tests and nutritional state (MNA) and insulin resistance (HOMA-IR) and blood glucose were assessed by Spearman's rank correlation coefficient.

All statistical tests were two-tailed and statistical significance was set at $p<0.05$. When appropriate, false discovery rate was controlled at $5 \%$ using the Benjamini-Hochberg method. All analyses were carried out using the SAS/STAT statistical package, release 9.4 (SAS Institute Inc., Cary, NC, USA).

\section{Results}

\subsection{Patients General Characteristics}

The study showed that AD patients (Table 1) had normal anthropometric measures (body weight; body mass index, BMI; mid-arm circumference [35]) and biohumoral variables, with a slight increase in the erythrosedimentation rate. These variables were not significantly different from those found in C-NNDS. AD patients presented with a state of insulin-resistance (HOMA-IR). Notwithstanding 
insulin resistance, the patients had normal glycosylated haemoglobin. Circulating proteins were mildly lower than normal values but albumin levels were still normal.

Table 1. Non-amino acid variables of the entire population of patients with Alzheimer's disease (AD) and of the control group (C-NNDS).

\begin{tabular}{|c|c|c|}
\hline Variable & $\begin{array}{l}\text { AD Patients } \\
\quad(N=54)\end{array}$ & $\begin{array}{l}\text { C-NNDS } \\
(N=17)\end{array}$ \\
\hline \multicolumn{3}{|l|}{ Demographic variables } \\
\hline Age (years) & $74.63 \pm 8.03$ & $71.8 \pm 6.3$ \\
\hline Male gender (\%) & $37(69 \%)$ & $7(41.2 \%)$ \\
\hline \multicolumn{3}{|l|}{ Anthropometric variables } \\
\hline Education (years) & $7.6 \pm 0.1$ & $9.8 \pm 5.3$ \\
\hline Body weight (kg) & $63.69 \pm 12.67$ & $69.1 \pm 8.5$ \\
\hline Height $(\mathrm{cm})$ & $161.16 \pm 9.75$ & $164 \pm 6.4$ \\
\hline Body mass index $\left(\mathrm{kg} / \mathrm{m}^{2}\right)$ & $24.66 \pm 4.71$ & $25.7 \pm 4.9$ \\
\hline Mid-arm circumference $(\mathrm{cm})$ & $26.01 \pm 2.83$ & $27.4 \pm 3.5$ \\
\hline \multicolumn{3}{|l|}{ Biohumoral variables } \\
\hline Glucose (NV 70-115 mg/dL) & $89.17 \pm 15.25$ & $95.2 \pm 10.4$ \\
\hline Insulin (NV 4-24 $\mu \mathrm{U} / \mathrm{mL})$ & $12.63 \pm 5.32$ & - \\
\hline HOMA-IR $(\mathrm{NV}<2.4)$ & $3.84 \pm 2.09$ & - \\
\hline Glycosylated haemoglobin (4.8-5.9\%) & $5.59 \pm 0.71$ & $5.3 \pm 0.9$ \\
\hline Total cholesterol $(\mathrm{NV}<200 \mathrm{mg} / \mathrm{dL})$ & $185.98 \pm 32.89$ & $200.3 \pm 41.5$ \\
\hline HDL cholesterol (NV: M > 55 mg/dL; F > 65 mg/dL) & $55.75 \pm 16.34$ & - \\
\hline LDL cholesterol $(\mathrm{NV}<100 \mathrm{mg} / \mathrm{dL})$ & $109.49 \pm 27.86$ & - \\
\hline Transferrin (NV 200-360 mg/dL) & $230.29 \pm 37.69$ & - \\
\hline Iron (NV 59-158 $\mu \mathrm{g} / \mathrm{dL})$ & $84.27 \pm 25.67$ & $75.6 \pm 19.5$ \\
\hline Tryglycerides (NV 0-200 mg/dL) & $101.51 \pm 39.32$ & $132.8 \pm 25.6$ \\
\hline Vitamin B12 (NV 191-663 pg/mL) & $321.50 \pm 147.19$ & $352.7 \pm 100.5$ \\
\hline Folate (NV $3.1-17.5 \mathrm{ng} / \mathrm{mL})$ & $6.49 \pm 3.11$ & $5.3 \pm 2.5$ \\
\hline Creatinine (NV: M 0.73-1.18 mg/dL; F 0.55-1.02 mg/dL) & $0.90 \pm 0.22$ & $0.95 \pm 0.16$ \\
\hline Total proteins (NV 6.6-8.7 g/dL) & $6.29 \pm 0.40$ & $6.9 \pm 0.7$ \\
\hline Albumin (NV 3500-5200 mg/dL) & $3696 \pm 317$ & $3802 \pm 358$ \\
\hline White blood cell count $\left(\mathrm{NV} 4.00-10.00 \times 10^{3} / \mu \mathrm{L}\right)$ & $6.34 \pm 1.48$ & $5.8 \pm 1.02$ \\
\hline Red blood cell count (NV: M 4.30-5.70 × 10 $\left./ \mu \mathrm{L} ; \mathrm{F} 3.80-5.20 \times 10^{6} / \mu \mathrm{L}\right)$ & $4.34 \pm 0.45$ & $4.5 \pm 0.6$ \\
\hline Haemoglobin (NV: M 13.2-17.3 g/dL; F 11.7-15.5 g/dL) & $13.14 \pm 1.24$ & $13.8 \pm 1.7$ \\
\hline Erythrosedimentation rate $(\mathrm{NV}<15 \mathrm{~mm} / 1 \mathrm{st} \mathrm{h})$ & $17.44 \pm 17.81$ & $15 \pm 6.5$ \\
\hline \multicolumn{3}{|l|}{ AD biomarker concentrations in CSF } \\
\hline $\operatorname{tau}(\mathrm{NV}<404 \mathrm{pg} / \mathrm{mL})$ & $453.89 \pm 408.61$ & - \\
\hline p-tau $(\mathrm{NV}<56.5 \mathrm{pg} / \mathrm{mL})$ & $74.91 \pm 35.76$ & - \\
\hline$\beta$-amyloid (NV > 599 pg/mL) & $555.33 \pm 359.23$ & - \\
\hline$\beta$-amyloid/tau $(\mathrm{NV}>1.6)$ & $2.22 \pm 2.73$ & - \\
\hline$\beta$-amyloid/p-tau (NV > 11.5) & $9.35 \pm 8.27$ & - \\
\hline \multicolumn{3}{|l|}{ Neurocognitive tests } \\
\hline $\begin{array}{c}\text { Mini Mental State Examination (MMSE }<24 \text { denotes } \\
\text { cognitive impairment) }\end{array}$ & $18.42 \pm 7.10$ & - \\
\hline Digit Span $(\mathrm{NV}<3.75)$ & $3.90 \pm 0.85$ & - \\
\hline Trail Making Test TMT-A (NV < 93") & $156.33 \pm 57.29$ & - \\
\hline Trail Making Test TMT-B (NV < 282") & $194.27 \pm 107.49$ & - \\
\hline
\end{tabular}

Data are expressed as mean \pm standard deviation except for gender, expressed as $N(\%)$. Abbreviations: $N V$, normal values; HOMA-IR, HOmeostasis Model Assessment-Insulin Resistance; CSF, cerebrospinal fluid.

According to MNA, 51.9\% patients (28/54) had a normal nutritional state, $33.3 \%(18 / 54)$ were at risk of malnutrition, and $14.8 \%(8 / 54)$ were malnourished. 


\subsection{Plasma AA Concentrations in AD Patients and in C-NNDS}

With regard to plasma AA concentrations, in comparison to C-NNDS, AD patients had 45\% (9/20) of altered individual AAs: 35\% were lower (aspartic acid, glutamic acid, histidine, asparagine, serine citrulline, taurine; $p<0.02$ ), 10\% were higher (3-methyl-histidine, 3-MHI, lysine; $p<0.001$ ). Moreover, the AD patients exhibited a lower content of Total AAs (TAAs; $p<0.035$ ), a trend towards higher EAA/TAA ratio $(p=0.057)$, and a lower ratio of BCAAs/EAAs $(p=0.004)$.

The distributions of the altered AAs in AD patients stratified for nutritional status were similar in the three subgroups: $25 \%$ in both normonourished and at risk of malnutrition patients, $20 \%$ in malnourished patients.

To investigate whether the AA alterations were attributable to the primary disease per se and not to altered nutrition, we compared C-NNDS with each MNA-stratified subgroup (Table 2) and observed that each patient subgroup, in comparison to C-NNDS, shared the same pattern of significantly altered AAs: lower aspartic acid and taurine, and higher 3-MHI. This indicated a pattern of perturbed AAs independent of patients' nutritional state.

Table 2. Differences in plasma amino acid levels $(\mu \mathrm{mol} / \mathrm{L})$ in control subjects (C-NNDS), malnourished patients with Alzheimer's disease (AD) (MNA Group 1), AD patients at risk of malnutrition (MNA Group 2), and normonourished AD patients (MNA Group 3). The table shows that the 3-nutritionally stratified AD subgroups, compared to C-NNDS, had 3 altered AAs in common: aspartic acid, taurine, and 3-methyl-histidine.

\begin{tabular}{|c|c|c|c|c|c|}
\hline Amino Acids & $\begin{array}{l}\text { C-NNDS } \\
(N=17)\end{array}$ & $\begin{array}{l}\text { AD Patients } \\
\text { MNA Group } 1 \\
\quad(N=8)\end{array}$ & $\begin{array}{l}\text { AD Patients } \\
\text { MNA Group } 2 \\
(N=18)\end{array}$ & $\begin{array}{l}\text { AD Patients } \\
\text { MNA Group } 3 \\
(N=28)\end{array}$ & $p$ Value \\
\hline Aspartic acid & $27.86 \pm 4.34$ & $8.88 \pm 5.59^{+}$ & $7.17 \pm 2.79 \ddagger$ & $8.86 \pm 6.80 \ddagger$ & $<0.0001$ \\
\hline Glutamic acid & $107.74 \pm 52.29$ & $64.63 \pm 19.18$ & $60.33 \pm 17.32^{+}$ & $68.68 \pm 17.70$ & 0.008 \\
\hline Histidine & $60.22 \pm 35.79$ & $69.63 \pm 8.93$ & $67.17 \pm 13.29$ & $71.39 \pm 14.14^{\wedge}$ & 0.025 \\
\hline Asparagine & $50.99 \pm 14.84$ & $40.25 \pm 4.30$ & $39.33 \pm 6.54$ & $42.71 \pm 6.51$ & 0.015 \\
\hline Serine & $130.79 \pm 27.39$ & $102.13 \pm 19.75$ & $96.56 \pm 22.57^{+}$ & $110.00 \pm 48.16^{\dagger}$ & 0.0009 \\
\hline Glutamine & $488.95 \pm 75.61$ & $515.00 \pm 82.56$ & $499.72 \pm 83.32$ & $511.07 \pm 82.65$ & 0.86 \\
\hline 3-methyl-histidine & $0.87 \pm 0.39$ & $3.25 \pm 1.97^{\dagger}$ & $3.66 \pm 1.98 \ddagger$ & $3.88 \pm 1.30 \ddagger$ & $<0.0001$ \\
\hline Arginine & $64.40 \pm 19.60$ & $71.75 \pm 16.44$ & $63.89 \pm 24.23$ & $63.79 \pm 22.33$ & 0.62 \\
\hline Citrulline & $39.22 \pm 7.33$ & $35.88 \pm 9.80$ & $33.44 \pm 8.94$ & $35.32 \pm 11.75$ & 0.15 \\
\hline Glycine & $224.84 \pm 61.68$ & $234.88 \pm 38.80$ & $246.11 \pm 93.85$ & $232.79 \pm 81.78$ & 0.81 \\
\hline Threonine & $129.30 \pm 42.21$ & $125.88 \pm 23.14$ & $116.56 \pm 28.67$ & $118.07 \pm 28.58$ & 0.80 \\
\hline Alanine & $370.89 \pm 79.26$ & $321.50 \pm 39.71$ & $371.67 \pm 91.31$ & $344.14 \pm 74.21$ & 0.29 \\
\hline Taurine & $106.93 \pm 32.71$ & $82.25 \pm 71.18^{+}$ & $71.72 \pm 25.77^{\wedge}$ & $64.39 \pm 15.10 \ddagger$ & $<0.0001$ \\
\hline Tyrosine & $59.21 \pm 17.61$ & $53.50 \pm 11.95$ & $54.00 \pm 13.56$ & $53.21 \pm 12.47$ & 0.65 \\
\hline Tryptophan & $47.23 \pm 12.37$ & $42.13 \pm 11.10$ & $44.22 \pm 10.80$ & $46.11 \pm 8.36$ & 0.47 \\
\hline Phenylalanine & $61.32 \pm 24.76$ & $59.63 \pm 23.54$ & $52.78 \pm 11.31$ & $53.11 \pm 10.19$ & 0.67 \\
\hline Isoleucine & $61.37 \pm 14.60$ & $57.38 \pm 14.19$ & $54.17 \pm 20.55$ & $60.00 \pm 14.70$ & 0.45 \\
\hline Leucine & $122.37 \pm 25.25$ & $105.38 \pm 35.32$ & $102.28 \pm 30.76$ & $116.79 \pm 24.39$ & 0.08 \\
\hline Lysine & $162.26 \pm 35.02$ & $219.25 \pm 59.62$ & $195.17 \pm 44.17$ & $205.21 \pm 28.65^{\dagger}$ & 0.003 \\
\hline Valine & $246.49 \pm 53.63$ & $205.48 \pm 68.88$ & $199.44 \pm 59.98^{\wedge}$ & $227.73 \pm 47.55$ & 0.046 \\
\hline TAAs & $2581.83 \pm 263.18$ & $2428.11 \pm 365.33$ & $2387.83 \pm 424.43$ & $2446.96 \pm 308.65$ & 0.27 \\
\hline BCAAs & $430.23 \pm 82.36$ & $368.23 \pm 117.08$ & $355.89 \pm 110.57$ & $404.52 \pm 84.87$ & 0.061 \\
\hline EAAs & $891.43 \pm 137.92$ & $887.98 \pm 223.29$ & $835.44 \pm 196.77$ & $902.29 \pm 145.03$ & 0.45 \\
\hline EAAs/TAAs & $0.34 \pm 0.03$ & $0.36 \pm 0.04$ & $0.35 \pm 0.03$ & $0.37 \pm 0.02$ & 0.033 \\
\hline BCAAs/TAAs & $0.17 \pm 0.02$ & $0.15 \pm 0.03$ & $0.15 \pm 0.03$ & $0.16 \pm 0.02$ & 0.036 \\
\hline BCAAs/EAAs & $0.48 \pm 0.05$ & $0.41 \pm 0.04^{\dagger}$ & $0.42 \pm 0.04^{\dagger}$ & $0.45 \pm 0.03$ & $<0.0001$ \\
\hline
\end{tabular}

: $p<0.05$ vs. C-NNDS; ${ }^{\dagger}: p<0.01$ vs. C-NNDS; ${ }^{\ddagger}: p<0.0001$ vs. C-NNDS. Data are expressed as mean \pm standard deviation. Reported $p$ values are for between-group comparisons (Kruskal-Wallis test). False discovery rate was controlled at $5 \%$ by the Benjamini-Hochberg method. Significant differences were followed up by post-hoc paired comparisons (Dunn-Sidak test). Abbreviations: MNA, mini Nutritional assessment; TAAs, total amino acids; BCAAs, branched chain amino acids; EAAs, essential amino acids. 
To better understand the contribution of the nutritional state to the altered circulating AAs, these substrates were analysed in the 3 patient subgroups. Compared to normonourished patients, the subjects at risk of malnutrition had lower levels of both BCAA/TAA $(p=0.038)$ and BCAA/EAA $(p=0.026)$ ratios, whereas malnourished patients displayed a lower BCAA/EAA $(p=0.035)$ ratio. No differences were found between patients at risk of malnutrition and malnourished patients. After pooling together these two subgroups (combined group: CG), CG compared to the normonourished subgroup (Table 3$)$ showed lower levels of leucine $(p=0.047)$, valine $(p=0.047)$, and tended to have lower BCAA levels $(p=0.06)$ and lower EAA/TAA- $(p=0.023)$, BCAA/TAA- $(p=0.009)$ and $\mathrm{BCAA} / \mathrm{EAA}(p=0.002)$ ratios.

Table 3. Levels of plasma amino acids $(\mu \mathrm{mol} / \mathrm{L})$ and statistical analysis between combined group (CG $=$ malnourished-MNA Group $1+$ at risk of malnutrition-MNA Group 2) and normonourished-MNA Group 3 patients according to Mini Nutritional Assessment (MNA).

\begin{tabular}{|c|c|c|c|}
\hline Amino Acids & $\begin{array}{c}C G \\
(N=26)\end{array}$ & $\begin{array}{c}\text { MNA Group } 3 \\
\quad(N=28)\end{array}$ & $p$ Value \\
\hline Aspartic acid & $7.69 \pm 3.83$ & $8.86 \pm 6.80$ & 0.63 \\
\hline Glutamic acid & $61.65 \pm 17.64$ & $68.68 \pm 17.70$ & 0.09 \\
\hline Histidine & $67.92 \pm 11.99$ & $71.39 \pm 14.14$ & 0.16 \\
\hline Asparagine & $39.62 \pm 5.87$ & $42.71 \pm 6.51$ & 0.10 \\
\hline Serine & $98.27 \pm 21.51$ & $110.00 \pm 48.16$ & 0.39 \\
\hline Glutamine & $504.42 \pm 81.73$ & $511.07 \pm 82.65$ & 0.67 \\
\hline 3-methyl-histidine & $3.53 \pm 1.95$ & $3.88 \pm 1.30$ & 0.37 \\
\hline Arginine & $66.31 \pm 22.10$ & $63.79 \pm 22.33$ & 0.59 \\
\hline Citrulline & $34.19 \pm 9.09$ & $35.32 \pm 11.75$ & 0.94 \\
\hline Glycine & $242.65 \pm 80.24$ & $232.79 \pm 81.78$ & 0.47 \\
\hline Threonine & $119.42 \pm 26.99$ & $118.07 \pm 28.58$ & 0.56 \\
\hline Alanine & $356.23 \pm 81.66$ & $344.14 \pm 74.21$ & 0.64 \\
\hline Taurine & $74.96 \pm 43.53$ & $64.39 \pm 15.10$ & 0.50 \\
\hline Tyrosine & $53.85 \pm 12.85$ & $53.21 \pm 12.47$ & 0.92 \\
\hline Tryptophan & $43.58 \pm 10.72$ & $46.11 \pm 8.36$ & 0.25 \\
\hline Phenylalanine & $54.88 \pm 15.89$ & $53.11 \pm 10.19$ & 0.73 \\
\hline Isoleucine & $55.15 \pm 18.60$ & $60.00 \pm 14.70$ & 0.30 \\
\hline Leucine & $103.23 \pm 31.54$ & $116.79 \pm 24.39$ & 0.047 \\
\hline Lysine & $202.58 \pm 49.50$ & $205.21 \pm 28.65$ & 0.48 \\
\hline Valine & $201.30 \pm 61.51$ & $227.73 \pm 47.55$ & 0.047 \\
\hline TAAs & $2400.22 \pm 400.28$ & $2446.96 \pm 308.65$ & 0.72 \\
\hline BCAAs & $359.68 \pm 110.39$ & $404.52 \pm 84.87$ & 0.060 \\
\hline EAAs & $851.60 \pm 202.24$ & $902.29 \pm 145.03$ & 0.17 \\
\hline EAAs/TAAs & $0.35 \pm 0.04$ & $0.37 \pm 0.02$ & $0.023^{\wedge}$ \\
\hline BCAAs/TAAs & $0.15 \pm 0.03$ & $0.16 \pm 0.02$ & $0.009^{\wedge}$ \\
\hline BCAAs/EAAs & $0.42 \pm 0.04$ & $0.45 \pm 0.03$ & $0.002^{\wedge}$ \\
\hline
\end{tabular}

Data are expressed as mean values \pm standard deviation. Reported $p$ values are for between group comparisons (Kruskal-Wallis test). Only $p$ values followed by the symbol remained significant after control for false discovery rate. Abbreviations: MNA, mini nutritional assessment; TAAs, total amino acids; BCAAs, branched chain amino acids; EAAs, essential amino acids.

Of interest, regarding the non-amino acid variables, after their stratification for patient MNA groups (Table 4), CG group (malnourished-MNA Group $1+$ at risk of malnutrition-MNA Group 2) in comparison to MNA Group 3 (normonourished AD patients) had significant reductions in body weight, red blood cell count, and haemoglobin concentration. 
Table 4. Non-amino acid variables of the population of patients with Alzheimer's disease (AD) subdivided into malnourished patients (MNA Group 1), patients at risk of malnutrition (MNA Group 2), normonourished patients (MNA Group 3), and combined group (CG = MNA Group 1 + MNA Group 2).

\begin{tabular}{|c|c|c|c|c|c|}
\hline Variable & $\begin{array}{c}\text { CG } \\
(N=26)\end{array}$ & $\begin{array}{l}\text { MNA Grp } 1 \\
\quad(N=8)\end{array}$ & $\begin{array}{l}\text { MNA Grp } 2 \\
(N=18)\end{array}$ & $\begin{array}{l}\text { MNA Grp } 3 \\
(N=28)\end{array}$ & $\begin{array}{l}p \text { Value CG } \\
\text { vs. Grp } 3\end{array}$ \\
\hline \multicolumn{6}{|l|}{ Demographic variables } \\
\hline Age (years) & $74.58 \pm 9.01$ & $75.38 \pm 5.42$ & $74.22 \pm 10.33$ & $74.32 \pm 7.11$ & 0.81 \\
\hline Male gender (\%) & $17(65 \%)$ & $5(62 \%)$ & $12(67 \%)$ & $20(71 \%)$ & 0.07 \\
\hline \multicolumn{6}{|l|}{ Anthropometric variables } \\
\hline Education (years) & $7.11 \pm 4.08$ & $8.33 \pm 4.59$ & $6.54 \pm 3.89$ & $7.41 \pm 3.75$ & 0.84 \\
\hline Body weight (kg) & $59.15 \pm 11.46$ & $55.70 \pm 13.42$ & $60.68 \pm 10.52$ & $68.11 \pm 12.83$ & 0.006 \\
\hline Height $(\mathrm{cm})$ & $158.42 \pm 10.82$ & $162.00 \pm 8.64$ & $156.83 \pm 11.52$ & $163.14 \pm 8.66$ & 0.10 \\
\hline Body mass index $\left(\mathrm{kg} / \mathrm{m}^{2}\right)$ & $23.76 \pm 5.00$ & $21.58 \pm 6.52$ & $24.73 \pm 4.00$ & $25.60 \pm 4.49$ & 0.09 \\
\hline Mid-arm circumference $(\mathrm{cm})$ & $25.56 \pm 3.24$ & $23.44 \pm 3.83$ & $26.50 \pm 2.53$ & $26.43 \pm 2.36$ & 0.34 \\
\hline \multicolumn{6}{|l|}{ Biohumoral variables } \\
\hline Glucose (NV 70-115 mg/dL) & $85.50 \pm 9.74$ & $86.00 \pm 5.93$ & $85.28 \pm 11.18$ & $90.36 \pm 14.56$ & 0.36 \\
\hline Insulin (NV 4-24 $\mu \mathrm{U} / \mathrm{mL})$ & $11.10 \pm 4.37$ & $9.89 \pm 3.37$ & $11.64 \pm 4.73$ & $13.52 \pm 5.53$ & 0.11 \\
\hline HOMA-IR (NV < 2.4) & $3.41 \pm 1.99$ & $2.55 \pm 1.07$ & $3.79 \pm 2.21$ & $4.07 \pm 1.89$ & 0.15 \\
\hline $\begin{array}{c}\text { Glycosylated haemoglobin } \\
(4.8-5.9 \%)\end{array}$ & $5.53 \pm 0.51$ & $5.58 \pm 0.21$ & $5.52 \pm 0.60$ & $5.61 \pm 0.88$ & 0.69 \\
\hline $\begin{array}{c}\text { Total cholesterol } \\
(\mathrm{NV}<200 \mathrm{mg} / \mathrm{dL})\end{array}$ & $188.77 \pm 32.44$ & $196.50 \pm 36.26$ & $185.33 \pm 31.08$ & $186.04 \pm 32.87$ & 0.96 \\
\hline \multicolumn{6}{|l|}{ HDL cholesterol (NV: } \\
\hline $\begin{array}{l}\mathrm{M}>55 \mathrm{mg} / \mathrm{dL} \\
\mathrm{F}>65 \mathrm{mg} / \mathrm{dL})\end{array}$ & $56.85 \pm 17.72$ & $52.88 \pm 12.53$ & $58.61 \pm 19.66$ & $53.68 \pm 14.05$ & 0.71 \\
\hline $\begin{array}{l}\text { LDL cholesterol } \\
(\mathrm{NV}<100 \mathrm{mg} / \mathrm{dL})\end{array}$ & $111.69 \pm 25.28$ & $123.25 \pm 23.43$ & $106.56 \pm 24.96$ & $110.25 \pm 26.96$ & 0.89 \\
\hline $\begin{array}{l}\text { Transferrin (NV } \\
\text { 200-360 mg/dL) }\end{array}$ & $230.69 \pm 33.89$ & $220.25 \pm 40.08$ & $235.33 \pm 30.88$ & $229.07 \pm 41.89$ & 0.72 \\
\hline 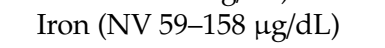 & $86.38 \pm 29.37$ & $91.13 \pm 26.63$ & $84.28 \pm 31.01$ & $83.43 \pm 22.24$ & 0.72 \\
\hline $\begin{array}{l}\text { Tryglycerides (NV } \\
0-200 \mathrm{mg} / \mathrm{dL})\end{array}$ & $99.00 \pm 34.58$ & $100.13 \pm 45.82$ & $98.50 \pm 29.89$ & $105.36 \pm 43.55$ & 0.94 \\
\hline $\begin{array}{l}\text { Vitamin B12 (NV } \\
191-663 \text { pg/mL) }\end{array}$ & $331.96 \pm 139.31$ & $446.88 \pm 150.31$ & $277.88 \pm 97.56$ & $318.00 \pm 162.25$ & 0.47 \\
\hline $\begin{array}{c}\text { Folate (NV 3.1-17.5 ng/mL) } \\
\text { Creatinine (NV: }\end{array}$ & $6.47 \pm 3.49$ & $6.21 \pm 3.66$ & $6.59 \pm 3.52$ & $6.64 \pm 2.93$ & 0.49 \\
\hline $\begin{array}{l}\text { M } 0.73-1.18 \mathrm{mg} / \mathrm{dL} ; \\
\text { F } 0.55-1.02 \mathrm{mg} / \mathrm{dL})\end{array}$ & $0.90 \pm 0.24$ & $0.97 \pm 0.33$ & $0.86 \pm 0.18$ & $0.91 \pm 0.21$ & 0.66 \\
\hline $\begin{array}{l}\text { Total proteins (NV } \\
6.6-8.7 \mathrm{~g} / \mathrm{dL})\end{array}$ & $6.33 \pm 0.49$ & $6.29 \pm 0.79$ & $6.35 \pm 0.30$ & $6.27 \pm 0.33$ & 0.79 \\
\hline $\begin{array}{c}\text { Albumin (NV } \\
3500-5200 \mathrm{mg} / \mathrm{dL})\end{array}$ & $3683.85 \pm 360.31$ & $3572.50 \pm 560.91$ & $3733.33 \pm 230.09$ & $3728.93 \pm 284.84$ & 0.54 \\
\hline $\begin{array}{l}\text { White blood cell count (NV } \\
\left.4.00-10.00 \times 10^{3} / \mu \mathrm{L}\right)\end{array}$ & $6.59 \pm 1.79$ & $7.16 \pm 2.12$ & $6.32 \pm 1.61$ & $6.11 \pm 1.21$ & 0.60 \\
\hline $\begin{array}{l}\text { Red blood cell count (NV: } \\
\text { M 4.30-5.70 × } 10^{6} / \mu \mathrm{L} ; \\
\left.\text { F 3.80-5.20 × } 10^{6} / \mu \mathrm{L}\right)\end{array}$ & $4.20 \pm 0.46$ & $4.21 \pm 0.41$ & $4.19 \pm 0.50$ & $4.47 \pm 0.44$ & 0.025 \\
\hline $\begin{array}{l}\text { Haemoglobin (NV: } \\
\text { M 13.2-17.3 g/dL; } \\
\text { F 11.7-15.5 g/dL) }\end{array}$ & $12.81 \pm 1.31$ & $12.70 \pm 0.95$ & $12.86 \pm 1.47$ & $13.44 \pm 1.18$ & 0.035 \\
\hline $\begin{array}{l}\text { Erythrosedimentation rate } \\
\quad(\mathrm{NV}<15 \mathrm{~mm} / 1 \mathrm{st} \mathrm{h})\end{array}$ & $23.28 \pm 22.19$ & $24.25 \pm 24.67$ & $22.82 \pm 21.71$ & $13.00 \pm 12.16$ & 0.07 \\
\hline $\begin{array}{l}\text { AD biomarkers in CSF } \\
\text { tau }(\mathrm{NV}<404 \mathrm{pg} / \mathrm{mL})\end{array}$ & $486.86 \pm 609.36$ & $231.60 \pm 107.46$ & $628.67 \pm 730.94$ & $444.14 \pm 216.02$ & 0.14 \\
\hline p-tau $(\mathrm{NV}<56.5 \mathrm{pg} / \mathrm{mL})$ & $72.07 \pm 44.15$ & $45.60 \pm 20.13$ & $86.78 \pm 47.78$ & $79.20 \pm 28.62$ & 0.28 \\
\hline $\begin{array}{c}\beta \text {-amyloid } \\
(\mathrm{NV}>599 \mathrm{pg} / \mathrm{mL})\end{array}$ & $532.29 \pm 471.59$ & $343.60 \pm 172.28$ & $637.11 \pm 558.52$ & $566.95 \pm 283.63$ & 0.18 \\
\hline$\beta$-amyloid/tau $(\mathrm{NV}>1.6)$ & $2.46 \pm 3.43$ & $1.94 \pm 1.35$ & $2.74 \pm 4.23$ & $2.02 \pm 2.31$ & 0.96 \\
\hline$\beta$-amyloid/p-tau $(\mathrm{NV}>11.5)$ & $9.92 \pm 10.33$ & $9.52 \pm 7.52$ & $10.14 \pm 12.04$ & $8.33 \pm 6.32$ & 0.99 \\
\hline
\end{tabular}


Table 4. Cont.

\begin{tabular}{|c|c|c|c|c|c|}
\hline Variable & $\begin{array}{c}\text { CG } \\
(N=26)\end{array}$ & $\begin{array}{l}\text { MNA Grp } 1 \\
\quad(N=8)\end{array}$ & $\begin{array}{l}\text { MNA Grp } 2 \\
(N=18)\end{array}$ & $\begin{array}{l}\text { MNA Grp } 3 \\
(N=28)\end{array}$ & $\begin{array}{l}p \text { Value CG } \\
\text { vs. Grp } 3\end{array}$ \\
\hline \multicolumn{6}{|l|}{ Neurocognitive tests } \\
\hline Mini Mental State & & & & & \\
\hline $\begin{array}{c}\text { Examination } \\
\text { (MMSE }<24 \text { denotes } \\
\text { cognitive impairment) }\end{array}$ & $15.75 \pm 7.30$ & $17.17 \pm 5.75$ & $15.11 \pm 8.07$ & $19.14 \pm 7.13$ & 0.20 \\
\hline Digit span $(\mathrm{NV}<3.75)$ & $4.06 \pm 0.77$ & $4.92 \pm 0.52$ & $3.63 \pm 0.41$ & $3.78 \pm 1.00$ & 0.52 \\
\hline $\begin{array}{l}\text { Trail Making Test TMT-A } \\
\qquad\left(\mathrm{NV}<93^{\prime \prime}\right)\end{array}$ & $162.00 \pm 56.19$ & $178.00 \pm 33.94$ & $155.60 \pm 65.34$ & $145.21 \pm 55.31$ & 0.65 \\
\hline $\begin{array}{l}\text { Trail Making Test TMT-B } \\
\qquad\left(\mathrm{NV}<282^{\prime \prime}\right)\end{array}$ & $262.00 \pm 136.96$ & $347.00 \pm 0.00$ & $219.50 \pm 163.34$ & $162.33 \pm 94.98$ & 0.24 \\
\hline
\end{tabular}

Data are expressed as mean \pm standard deviation except for gender, expressed as $N(\%)$. Reported $p$ values are for between-group comparison (CG vs. MNA Group 3, Kruskal-Wallis test). Abbreviations: MNA, Mini Nutritional Assessment; NV, normal values; HOMA-IR, HOmeostasis Model Assessment-Insulin Resistance; CSF, cerebrospinal fluid.

Thus, we found that HOMA-IR was similar in the various subgroups.

\subsection{Correlations between Plasma AAs and CSF Concentrations of AD Biomarkers}

The study showed several significant correlations between plasma AAs and CSF concentrations of AD biomarkers.

$\mathrm{A} \beta$ was negatively associated with histidine $(r=-0.33, p<0.05)$. p-tau was positively correlated with serine $(r=+0.40, p<0.05)$ and especially glycine $(r=+0.63, p<0.001)$. Tau was positively linked to aspartate $(r=+0.33, p<0.05)$, serine $(r=+0.39, p<0.05)$, alanine $(r=+0.45, p<0.01)$ and glycine $(r=+0.55, p<0.001)$.

To sum up, the results showed that: (1) significant alterations in plasma levels of aspartic acid, taurine, and 3-MHI were present in all patients' nutritional categories, indicating that they were constitutive of the primary disease; (2) alterations in BCAAs/TAAs, EAAs/TAAs, and BCAAs/EAAs seem to be associated with patients' nutritional state, showing a tendency to be lower in the CG group than in normonourished $\mathrm{AD}$ patients.

\subsection{Correlations between Non-Amino Acid Variables and Respectively Plasma AAs with Neurocognitive Tests}

The study found that the neurocognitive tests were similar in the 3 patient subgroups and showed no significant correlation with nutritional state (MNA) $(r$ between MNA and MMSE $=+0.20$, Digit Span $r=-0.18$, TMT-A $r=-0.02$, TMT-B $r=-0.25$ ).

Cognitive impairment (MMSE < 24) was observed in $78 \%$ of the patients $(80,91$, and $71 \%$, in MNA Group 1, 2, and 3, respectively), Digit Span exceeded NV value in 38\% of the patients (60, 27 and 38\% in MNA Group 1, 2, and 3, respectively), TMT-A exceeded NV value in $43 \%$ of the patients $(40 \%, 36 \%$, and $46 \%$ in MNA Group 1, 2, and 3, respectively) and TMT-B exceeded NV value in $8 \%$ of the patients (20\%, $9 \%$, and $4 \%$ in MNA Group 1, 2, and 3, respectively).

Moreover, no significant associations were found between neurocognitive tests and insulin resistance (HOMA-IR) ( $r$ HOMA-IR vs. MMSE $=-0.02$, Digit Span $r=+0.08$, TMT-A $r=+0.10$, TMT-B $r=+0.23$ ) nor between neurocognitive tests and blood glucose ( $r$ blood glucose vs. MMSE $=+0.15$, Digit Span $r=-0.21$, TMT-A $r=-0.09$, TMT-B $r=-0.23$ ).

Furthermore, plasma tryglycerides and body weight were negatively correlated with short-term verbal memory (Digit Span; $r=-0.43$ and respectively $r=-0.47, p<0.05$ ). Serum transferrin was negatively associated with TMT-B $(r=-0.68, p<0.05)$. Both serum vitamin B12 and total proteins concentrations were negatively associated $(r=-0.47$ and respectively $r=-0.49, p<0.05$ for both) with TMT-A.

With respect to plasma AAs and neurocognitive tests, serine levels were positively associated with Digit Span $(r=+0.34, p<0.05)$. TAAs were negatively associated with both TMT-A $(r=-0.43$, 
$p<0.05)$ and TMT-B $(r=-0.55, p<0.05)$. Histidine levels were positively correlated with TMT-A $(r=+0.44, p<0.05)$ and with TMT-B $(r=+0.82, p<0.01)$.

No significant correlations between the other AAs and neurocognitive tests were observed.

\section{Discussion}

The results of the study are in line with our initial hypothesis that AD patients may have two different patterns of altered circulating AAs, one due to the primary disease per se, the other likely associated with nutritional conditions. Moreover, the study shows that some circulating AAs correlate with neurocognitive tests such as short-term verbal memory and attention deficits.

The combination of low levels of both aspartate and taurine with higher 3-MHI seems to be constitutive of the AD per se whereas a higher EAA/TAA ratio, a lower BCAA/EAA ratio, and a trend towards lower BCAA levels seem linked to the deterioration of nutritional state.

This research suggests that taking into account patients' nutritional states may be useful to differentiate the alterations of AAs due to AD from those linked to patients' nutritional states. Thus, we will only discuss the AA alterations due to $\mathrm{AD}$ per se and those linked to patients' nutritional state.

\subsection{AA Alterations Linked to AD Per Se}

The combination of the two patterns might reconcile the discrepancies in plasma AAs found in previous studies [5,36]. On the other hand, discrepant plasma AA profiles in previous studies should not be surprising because the metabolic conditions of AD patients may be greatly heterogeneous as they are dependent not only on biochemical alterations due to A $\beta$ deposition [37] but also on patients' protein intake, body muscle proteolysis [38], insulin resistance [17], and severe muscle and brain metabolic derangements [37]. In addition, the overtime changes of these factors can further modify plasma AA contents and profiles. The interplay of all these factors may alter patients' nutritional state.

In the current investigation, altered $\mathrm{AA}$ levels due to $\mathrm{AD}$ per se may reflect intracellular metabolic disturbances following $A \beta$ deposition such as (1) difficulties in muscle energy formation leading to increased consumption of aspartic acid, a substrate that plays a key role both in anaerobic and aerobic pathways [37]; (2) muscle hypercatabolism (3-MHI); (3) increased consumption of antioxidant systems leading to low taurine [37]. Muscle hypercatabolism leads to increased AA release that may explain the higher EAA/TAA ratio in AD.

Aspartic acid plays a major role in the production of high energy phosphate compounds (ATP) as it forms the malate-aspartate cycle, which is not only the main mechanism for the oxidation of glucose and lactate [39], but it also regulates the entry of carbon skeletons from AAs into the tricarboxylic chain acid (TCA) cycle [40]. We postulate that in the study population, low plasma aspartic acid levels may be due to unbalanced high ratio cell consumption/diet provision + endogenous formation.

It is noteworthy that higher lysine concentrations in the overall AD population may confirm both the difficulty in muscle aerobic energy formation [5] and impaired protein synthesis [22]. Indeed, lysine, an essential AA, is only used for protein synthesis and is a precursor of carnitine formation, a substance essential for fat acid entry into mitochondria to be oxidised.

Low taurine may be caused by low availability of its precursors, methionine and cysteine. In this study, however, this was not the case, as the levels of taurine precursors were normal. Both inflammation and oxidative stress are likely to explain the low taurine [41,42]. As taurine has antioxidant properties, its low availability might aggravate oxidative stress and intracellular and mitochondrial overload [43].

Elevated 3-MHI reflects hypercatabolism of muscle contractile proteins [44]. Therefore, alterations in plasma AAs linked to the primary disease per se suggest that patients with $A D$, even if normonourished, have an increased risk of or are developing muscle wasting due to energy deficits and myofibrillar protein catabolism [37]. 


\subsection{Correlations between Plasma AAs and CSF Concentrations of AD Biomarkers}

The current study cannot explain the associations found between several plasma AAs and the CSF concentrations of the histological hallmarks of AD. We can only formulate some hypotheses.

Future studies targeting contemporary determinations of peripheral and CSF AA concentrations might help explain the mechanisms of these associations.

An excess of plasma histidine, an aromatic AA, may further reduce the BCAA/aromatic AA ratio at the BBB and may limit the BCAA entry to the brain. Reduced brain BCAA availability might reduce the reuptake of interstitial excitotoxic glutamate [45], favouring, in turn, neurodegeneration, increased $\mathrm{A} \beta$ formation leading to imbalanced ratio between production and clearance of the $\mathrm{A} \beta$ peptide [46].

The important correlation of glycine and serine (which can be converted to glycine) with p-tau protein could be explained by glycine (and glutamate) activation of NMDA receptors [47-49]. In AD, NMDA receptors are continuously activated [50].

The link between plasma aspartate and p-tau could be due to aspartate stimulation of the NMDA receptors with subsequent neurotoxicity. Of note, plasma aspartate and glycine are physiologically restricted in their entry to the brain.

Alanine is produced by the BCAA leucine metabolism. The positive correlation between plasma alanine and CSF tau levels could be due to both a possible diversion of leucine from the glutamate/glutamine cycle and to the reduced astrocyte uptake of interstitial excitotoxic glutamate, which is an important factor for neurodegeneration.

\subsection{Neurocognitive Tests and Patients' General Characteristics}

The study suggests that in AD patients, short-term verbal memory is associated with increases in both tryglyceride levels and body weight. Even though correlation does not necessarily mean a cause-effect relationship, the results are supported by at least two studies, one documenting that short term consumption of a high fat diet by aged animals leads to neuroinflammation and memory deficits [51], the other highlighting that obesity causes impairments of declarative memory [52].

Attention capacity deficits are lower with increased serum levels of vitamin B12, total proteins, and transferrin. Results of both clinical and observational studies reported that vitamin B12 plays an important role in cognition [53]. Given that vitamin B12 is connected with mitochondrial function, myelin integrity, and oxidative stress (and peripheral nerve function) [54], the lack of the vitamin may damage the brain structures deputed to cognitive functions. The inverse relationships between attention capacity deficits, on one hand, and circulating total proteins, transferrin, on the other hand, may account for the beneficial impact of the body/brain protein and AA metabolism and the transferrin modulation of iron transport to the brain. In fact, transferrin is a complex protein system that chelates iron. This metal is involved in brain function and dopaminergic activity. Increasing dopamine signalling, for example, by methylphenidate, is the first-line stimulant treatment for attention deficit/hyperactivity disorder [55]. It is interesting to note that in the study patients, the negative correlations of transferrin with attention capacity deficits seem to be in contrast to the role played by transferrin in mediating the effect of iron on the production and the deposition of $A \beta$, thus contributing to the pathogenesis of $\mathrm{AD}[56]$.

\subsection{AA Alterations Linked to Nutritional State}

With respect to plasma AAs linked to patients' nutritional state, the patients at risk for malnutrition and those with malnutrition as a combined group (CG), when compared to normonourished AD patients, had a tendency towards lower ratios of EAAs/TAAs, BCAAs/TAAs, and BCAAs/EAAs. It is of note that these AA differences occurred notwithstanding the non-amino acid variable differences between CG and normonourished AD patients. The differences in AA ratios indicate an overconsumption of EAAs, in particular BCAAs, which is not adequately compensated for by diet EAA intakes. Likely, BCAAs were mainly used to produce energy within skeletal muscles, as these AAs represent the preferential 
substrates for energy production. This is supported by a low BCAA/EAA ratio. Had BCAAs mainly been used for protein synthesis, we would have observed a more balanced BCAA/EAA ratio [18].

Besides their nutritional activities, BCAAs play a crucial role for brain function. These AAs are easily taken up by the brain [57] crossing the BBB in competition for the same transporter with the aromatic AAs phenylalanine, tyrosine and tryptophan [1]. In brain tissue, BCAAs are more catabolised than incorporated into proteins [58]. The first step in the catabolism of BCAAs is the reversible transamination with $\alpha$-ketoglutarate to produce the neurotransmitter glutamate and branched-chain $\alpha$-ketoacids (BCKAs). The reaction is catalysed by the branched-chain aminotransferase isozymes (BCAT), cytosolic BCATc [59], and mitochondrial BCATm [60]. In the brain, BCATc explains $70 \%$ of total BCAT activity [61]. In the brain, BCAAs provide amino groups of the synthesis of glutamate [62], and BCATc takes part in the glutamate/glutamine cycle [63]. A derivative of BCAA-formed excitatory glutamate is the inhibitory $\gamma$-aminobutyric acid (GABA).

Importantly, BCATc is present in neurons that use a variety of neurotransmitters such as cerebral cortex, white matter, internal capsule, globus pallidus, diencephalon, hypothalamus, tuberomammillary nucleus, many midbrain and brainstem nuclei, and spinal cord (grey matter) [45].

A recent research study has documented that blood BCAT proteins were increased in patients with $\mathrm{AD}$ in comparison to healthy controls [8]. Blood BCAT proteins and glutamate increase the sensitivity and specificity of Montreal Cognitive Assessment in correctly discriminating healthy subjects and subjects with AD, which is useful for an early diagnosis of AD [8]. The increase in blood BCAT paralleled the increased levels of BCATs found previously in the hippocampus, frontal and temporal cortex, in a post-mortem analysis of AD patients [64]. In this study, BCATm correlated with disease severity (Braak stage).

The importance of BCAAs has been documented in healthy subjects, in clinical and rehabilitation settings [8]. Supplementation of BCAAs improved cognitive performance in healthy subjects [65-68].

In the clinical setting, supplementation of BCAAs improved cognitive functions in patients with cirrhosis and chronic hepatic encephalopathy [69], in subjects with phenylketonuria [70]. In the rehabilitation ward, intravenous infusion of BCAAs enhanced the cognitive recovery of patients with severe traumatic brain injury [71] and improved recovery from a vegetative or minimally conscious state in patients following traumatic brain injury [72]. BCAA-induced cognitive recovery was subsequently documented in both an experimental model of traumatic brain injury [73] and in humans [74,75].

EAA consumption, when not adequately exogenously compensated, reduces metabolic support not only to muscles but also to vital organs including the brain and the heart [32]. EAA consumption may explain the associations between dementia and nutritional factors, such as the relationships between weight loss and dementia severity and faster progression [22], creatinine and dementia [9], and reduced BMI and medial temporal lobe atrophy [22]. Weight loss has been found to precede cognitive symptoms in AD [76]. We postulate that the higher EAA/TAA ratio that was found in the AD population compared to C-NNDS and the lower EAA/TAA ratio that was found in the AD CG group compared to normonourished AD may reflect two distinct but linked metabolic processes, the former an increase in muscle EAA release, the latter a body EAA overconsumption and/or reduced muscle mass, hence protein/AA content. If this hypothesis is correct, normonourished AD would have an excess of muscle EAA release, whereas non-normonourished AD may have EAA body overconsumption and/or reduced muscle mass.

In AD patients, reduced brain availability in EAAs and BCAAs may aggravate brain energy deficit [77], chemical neurotransmission, synaptic loss and synaptic malfunction [23], and glutamate neurotoxicity [45].

Recent research [9] has documented, in a large cohort of subjects, an association between low levels of BCAAs and increased risk of dementia, independently of conventional risk factors.

In the current study, the presence of perturbed EAA and BCAA levels in AD subjects who are at risk of malnutrition or malnourished might contribute to explaining the association between a lower MNA score and progression to dementia [76]. 
In the present investigation, the influence of circulating AAs on brain functioning may be witnessed by the associations of some AAs with neurocognitive tests. In a previous study, plasma glutamate correlated with visuo-spatial deficits [2]. However, we found a positive correlation between plasma serine levels and short-term verbal memory, and negative correlations between TAAs and attention deficits. Plasma histidine was positively correlated with attention deficits.

Circulating serine in the brain can be transferred to the stereoisomer D-serine, a potent activator of NMDA glutamate receptor, involved in the formation of new synapses which are important for learning and memory [78].

Notably, patients' attention capacity is correlated with TAAs and not with a single AA, which suggests the importance for brain function of optimal availability in all the AAs. It is more difficult to explain the positive relationship between plasma histidine and attention deficits, given that it has been reported that subjects affected by attention deficit disorder have significantly lower levels of histidine [79]. As histidine is an aromatic AA, an excess of this AA may limit the entry of phenylalanine and tyrosine into the brain, thus negatively influencing dopaminergic neurotransmission that plays a major role in attention capacity [80].

In the present study, the importance of circulating AAs for brain functioning is also witnessed by the absence of significance of the correlations between neurocognitive tests and HOMA-IR, neurocognitive tests and nutritional state (MNA), and neurocognitive tests and blood glucose. This means that at the disease stage in the study patients, the brain may be more susceptible to AAs than to glucose.

Interestingly, in the study, the AAs which correlated with the cognitive tests were those linked to patients' nutritional state and not to AD per se, suggesting that diet manipulation or appropriate AA supplementation may benefit $\mathrm{AD}$ patients with attentive dysfunction.

\section{Strengths and Limitations of the Study}

The strength of the current study is that administering a simple, clinical nutritional assessment test to AD patients may help physicians to identify patients' needs for essential and non-essential AAs. Moreover, the study reveals that low plasma levels of aspartate and taurine together with high plasma levels of 3-MHI seem to be constitutive of AD per se.

This study has several limitations that may be overcome by performing well-planned further investigations.

The findings of the study should be confirmed in a larger cohort of patients enrolled in different AD settings. The confirmation of altered AAs in AD including aspartic acid, 3-MHI, taurine, and serine is important for clinical practice because altered AAs can be exogenously manipulated.

Patients' nutritional state would have been better defined if we had measured body composition. For example, possible impairment of skeletal muscle mass would have allowed us to better understand the mechanisms underlying EAA/TAA ratio. However, the adoption of a simple nutritional test (MNA) may prove useful in a clinical setting to suggest aberrant plasma AA profiles before the occurrence of changes in standard nutrition variables (body weight, albumin levels) [76].

Measurements of AA levels in arterial blood would have allowed us to calculate the AA artero-venous differences and the net muscle release/uptake/no changes of AAs, thus providing more information about skeletal muscle protein/AA metabolism.

The determination of AAs both in plasma and CSF would have allowed us to better understand the possible utilisation of AAs in brain structures.

Patients were not requested to keep a food diary when at home. Analysing patients' nutritional intakes, mainly the amount and quality of ingested proteins, would have allowed us to have a better understanding of the interplay between AA intake and body/muscle protein metabolism.

Future studies will describe the relationships between plasma AAs and cognitive tests that have not been discussed in this research. 


\section{Suggestions for Clinical Practice and Future Directions}

This study provides some useful suggestions for both clinical practice and future investigations, addressing the effects on $\mathrm{AD}$ patients of the corrections of the aberrant AA profiles.

- In addition to altered AAs due to $\mathrm{AD}$ per se, subjects classified as at risk of or with malnutrition may be at high risk for overtime deterioration of EAAs, especially BCAAs. In these subjects, it would be prudent to monitor the adequacy of nutritional intake, in particular the amounts of both protein quantity and quality. In case of insufficient protein intake, the patients should be supplied with free EAAs/proteins.

- Future studies are needed to explore whether the recovery of aberrant plasma AAs could be relevant to AD subjects not only to maintain or improve the nutritional state but also to positively impact on neurocognition. We hypothesised that for these aims, combined supplemented proteins/AAs and exercise training might improve skeletal muscle mass, the main body tissue of AA store, circulating AAs, and brain availability.

\section{Conclusions}

The study shows that AD patients may be characterised by two altered patterns of plasma AA levels, one due to AD per se and the other likely associated with patients' nutritional state. Furthermore, the study found the existence of correlations between some AA levels and verbal memory and attention deficits.

Author Contributions: Conceptualisation, R.A.; methodology, M.C.R., M.B., A.P., M.V.; software, A.M.C.; validation, R.A., R.M., M.V.; formal analysis, R.M., M.B., A.P., V.T.; investigation, P.B., A.C., M.C., M.C.R.; resources, S.B.S.; data curation, R.M., M.B., A.P.; writing-original draft preparation, R.A.; writing-review and editing, M.V.; visualisation, F.B., D.B.; supervision, R.A., M.D.; project administration, R.A.; funding acquisition, M.D. All authors have read and agreed to the published version of the manuscript.

Funding: This research received no external funding.

Acknowledgments: This research was supported by the Italian Ministry of Education, University and Research (MIUR), Dipartimenti di Eccellenza Program (2018-2022)-Dept. of Biology and Biotechnology "L. Spallanzani", University of Pavia (to R.A., M.D., D.B., M.V.)

Conflicts of Interest: The authors declare no conflict of interest.

\section{References}

1. Fernstrom, J.D. Role of precursor availability in control of monoamine biosynthesis in brain. Physiol. Rev. 1983, 63, 484-546. [CrossRef] [PubMed]

2. Basun, H.; Forssell, L.G.; Almkvist, O.; Cowburn, R.F.; Eklöf, R.; Winblad, B.; Wetterberg, L.J. Amino acid concentrations in cerebrospinal fluid and plasma in Alzheimer's disease and healthy control subjects. Neural. Transm. Park. Dis. Dement. Sect. 1990, 2, 295-304. [CrossRef] [PubMed]

3. Kaiser, E.; Schoenknecht, P.; Kassner, S.; Hildebrandt, W.; Kinscherf, R.; Schroeder, J. Cerebrospinal fluid concentrations of functionally important amino acids and metabolic compounds in patients with mild cognitive impairment and Alzheimer's disease. Neurodegener. Dis. 2010, 7, 251-259. [CrossRef]

4. Olazarán, J.; Gil-de-Gómez, L.; Rodríguez-Martín, A.; Valentí-Soler, M.; Frades-Payo, B.; Marín-Muñoz, J.; Antúnez, C.; Frank-García, A.; Acedo-Jiménez, C.; Morlán-Gracia, L.; et al. A blood-based, 7-metabolite signature for the early diagnosis of Alzheimer's disease. J. Alzheimers Dis. 2015, 45, 1157-1173. [CrossRef] [PubMed]

5. Fonteh, A.N.; Harrington, R.J.; Tsai, A.; Liao, P.; Harrington, M.G. Free amino acid and dipeptide changes in the body fluids from Alzheimer's disease subjects. Amino Acids 2007, 32, 213-224. [CrossRef]

6. Ravaglia, G.; Forti, P.; Maioli, F.; Bianchi, G.; Martelli, M.; Talerico, T.; Servadei, L.; Zoli, M.; Mariani, E. Plasma amino acid concentrations in patients with amnestic mild cognitive impairment or Alzheimer disease. Am. J. Clin. Nutr. 2004, 80, 483-488. [CrossRef] 
7. Corso, G.; Cristofano, A.; Sapere, N.; la Marca, G.; Angiolillo, A.; Vitale, M.; Fratangelo, R.; Lombardi, T.; Porcile, C.; Intrieri, M.; et al. Serum amino acid profiles in normal subjects and in patients with or at risk of Alzheimer dementia. Dement. Geriatr. Cogn. Dis. Extra 2017, 7, 143-159. [CrossRef]

8. Hudd, F.; Shiel, A.; Harris, M.; Bowdler, P.; McCann, B.; Tsivos, D.; Wearn, A.; Knight, M.; Kauppinen, R.; Coulthard, E.; et al. Novel blood biomarkers that correlate with cognitive performance and hippocampal volumetry: Potential for early diagnosis of Alzheimer's disease. J. Alzheimers Dis. 2019, 67, 931-947. [CrossRef]

9. Tynkkynen, J.; Chouraki, V.; van der Lee, S.J.; Hernesniemi, J.; Yang, Q.; Li, S.; Beiser, A.; Larson, M.G.; Sääksjärvi, K.; Shipley, M.J.; et al. Association of branched-chain amino acids and other circulating metabolites with risk of incident dementia and Alzheimer's disease: A prospective study in eight cohorts. Alzheimers Dement. 2018, 14, 723-733. [CrossRef]

10. Toledo, J.B.; Arnold, M.; Kastenmüller, G.; Chang, R.; Baillie, R.A.; Han, X.; Thambisetty, M.; Tenenbaum, J.D.; Suhre, K.; Thompson, J.W.; et al. Metabolic network failures in Alzheimer's disease: A biochemical road map. Alzheimers Dement. 2017, 13, 965-984. [CrossRef]

11. Ibáñez, C.; Simó, C.; Martín-Álvarez, P.J.; Kivipelto, M.; Winblad, B.; Cedazo-Mínguez, A.; Cifuentes, A. Toward a predictive model of Alzheimer's disease progression using capillary electrophoresis-mass spectrometry metabolomics. Anal. Chem. 2012, 84, 8532-8540. [CrossRef] [PubMed]

12. Morifuji, M.; Ishizaka, M.; Baba, S.; Fukuda, K.; Matsumoto, H.; Koga, J.; Kanegae, M.; Higuchi, M. Comparison of different sources and degrees of hydrolysis of dietary protein: Effect on plasma amino acids, dipeptides, and insulin responses in human subjects. J. Agric. Food Chem. 2010, 58, 8788-8797. [CrossRef] [PubMed]

13. Hammer, J.A., 3rd; Rannels, D.E. Protein turnover in pulmonary macrophages. Utilization of amino acids derived from protein degradation. Biochem. J. 1981, 198, 53-65. [CrossRef] [PubMed]

14. Calsolaro, V.; Edison, P. Neuroinflammation in Alzheimer's disease: Current evidence and future directions. Alzheimers Dement. 2016, 12, 719-732. [CrossRef]

15. Shoemark, D.K.; Allen, S.J. The microbiome and disease: Reviewing the links between the oral microbiome, aging, and Alzheimer's disease. J. Alzheimers Dis. 2015, 43, 725-738. [CrossRef] [PubMed]

16. Liu, P.; Wu, L.; Peng, G.; Han, Y.; Tang, R.; Ge, J.; Zhang, L.; Jia, L.; Yue, S.; Zhou, K.; et al. Altered microbiomes distinguish Alzheimer's disease from amnestic mild cognitive impairment and health in a chinese cohort. Brain Behav. Immun. 2019, 80, 633-643. [CrossRef]

17. Drábková, P.; Šanderová, J.; Kovařík, J.; Kand'ár, R. An assay of selected serum amino acids in patients with type 2 diabetes mellitus. Adv. Clin. Exp. Med. 2015, 24, 447-451. [CrossRef]

18. Aquilani, R.; Brugnatelli, S.; Dossena, M.; Maestri, R.; Delfanti, S.; Buonocore, D.; Boschi, F.; Simeti, E.; Condino, A.M.; Verri, M. Oxaliplatin-fluoropyrimidine combination (XELOX) therapy does not affect plasma amino acid levels and plasma markers of oxidative stress in colorectal cancer surgery patients: A pilot study. Nutrients 2019, 11, E2667. [CrossRef]

19. Kuo, Y.M.; Kokjohn, T.A.; Watson, M.D.; Woods, A.S.; Cotter, R.J.; Sue, L.I.; Kalback, W.M.; Emmerling, M.R.; Beach, T.G.; Roher, A.E. Elevated abeta42 in skeletal muscle of Alzheimer disease patients suggests peripheral alterations of AbetaPP metabolism. Am. J. Pathol. 2000, 156, 797-805. [CrossRef]

20. Mukhamedyarov, M.A.; Teplov, A.Y.; Grishin, S.N.; Leushina, A.V.; Zefirov, A.L.; Palotás, A. Extraneuronal toxicity of Alzheimer's $\beta$-amyloid peptide: Comparative study on vertebrate skeletal muscles. Muscle Nerve 2011, 43, 872-877. [CrossRef]

21. Verri, M.; Aquilani, R.; Ricevuti, G.; Rondanelli, M.; Ghitti, M.; Bongiorno, A.I.; Venturini, L.; Buonocore, D.; Boschi, F.; Dossena, M. Plasma energy substrates at two stages of Alzheimer's disease in humans. Int. J. Immunopathol. Pharmacol. 2018, 32. [CrossRef]

22. Burns, J.M.; Johnson, D.K.; Watts, A.; Swerdlow, R.H.; Brooks, W.M. Reduced lean mass in early Alzheimer disease and its association with brain atrophy. Arch. Neurol. 2010, 67, 428-433. [CrossRef] [PubMed]

23. Liu, Y.; Li, N.; Zhou, L.; Li, Q.; Li, W. Plasma metabolic profiling of mild cognitive impairment and Alzheimer's disease using liquid chromatography/mass spectrometry. Cent. Nerv. Syst. Agents Med. Chem. 2014, 14, 113-120. [CrossRef] [PubMed]

24. Dubois, B.; Feldman, H.H.; Jacova, C.; Hampel, H.; Molinuevo, J.L.; Blennow, K.; DeKosky, S.T.; Gauthier, S.; Selkoe, D.; Bateman, R.; et al. Advancing research diagnostic criteria for Alzheimer's disease: The IWG-2 criteria. Lancet Neurol. 2014, 13, 614-629. [CrossRef] 
25. Parnetti, L.; Chiasserini, D.; Eusebi, P.; Giannandrea, D.; Bellomo, G.; De Carlo, C.; Padiglioni, C.; Mastrocola, S.; Lisetti, V.; Calabresi, P. Performance of $\mathrm{A} \beta_{1-40}, \mathrm{~A} \beta_{1-42}$, total tau, and phosphorylated tau as predictors of dementia in a cohort of patients with mild cognitive impairment. J. Alzheimers Dis. 2012, 29, 229-238. [CrossRef]

26. Albert, M.S.; DeKosky, S.T.; Dickson, D.; Dubois, B.; Feldman, H.H.; Fox, N.C.; Gamst, A.; Holtzman, D.M.; Jagust, W.J.; Petersen, R.C.; et al. The diagnosis of mild cognitive impairment due to Alzheimer's disease: Recommendations from the National Institute on Aging-Alzheimer's Association Workgroups on Diagnostic Guidelines for Alzheimer's disease. Alzheimers Dement. 2011, 7, 270-279. [CrossRef]

27. Ferris, S.H.; Yan, B. Differential diagnosis and clinical assessment of patients with severe Alzheimer disease. Alzheimer Dis. Assoc. Disord. 2003, 17, S92-S95. [CrossRef]

28. Vellas, B.; Guigoz, Y.; Garry, P.J.; Nourhashemi, F.; Bennahum, D.; Lauque, S.; Albarede, J.L. The Mini Nutritional Assessment (MNA) and its use in grading the nutritional state of elderly patients. Nutrition 1999, 15, 116-122. [CrossRef]

29. Spinnler, H.; Tognoni, G. Standardizzazione e taratura italiana di test neuropsicologici. Ital. J. Neurol. Sci. $1987,8,1-120$.

30. Giovagnoli, A.R.; Del Pesce, M.; Mascheroni, S.; Simoncelli, M.; Laiacona, M.; Capitani, E. Trail making test: Normative values from 287 normal adult controls. Ital. J. Neurol. Sci. 1996, 17, 305-309. [CrossRef]

31. Crowe, S.F. The differential contribution of mental tracking, cognitive flexibility, visual search, and motor speed to performance on parts A and B of the Trail Making Test. J. Clin. Psychol. 1998, 54, 585-591. [CrossRef]

32. Aquilani, R.; La Rovere, M.T.; Corbellini, D.; Pasini, E.; Verri, M.; Barbieri, A.; Condino, A.M.; Boschi, F. Plasma amino acid abnormalities in chronic heart failure. Mechanisms, potential risks and targets in human myocardium metabolism. Nutrients 2017, 9, 1251. [CrossRef] [PubMed]

33. Matthews, D.R.; Hosker, J.P.; Rudenski, A.S.; Naylor, B.A.; Treacher, D.F.; Turner, R.C. Homeostasis model assessment: Insulin resistance and beta-cell function from fasting plasma glucose and insulin concentrations in man. Diabetologia 1985, 28, 412-419. [CrossRef]

34. Solerte, S.B.; Gazzaruso, C.; Schifino, N.; Locatelli, E.; Destro, T.; Ceresini, G.; Ferrari, E.; Fioravanti, M. Metabolic effects of orally administered amino acid mixture in elderly subjects with poorly controlled type 2 diabetes mellitus. Am. J. Cardiol. 2004, 93, 23A-29A. [CrossRef] [PubMed]

35. Perissinotto, E.; Pisent, C.; Sergi, G.; Grigoletto, F.; ILSA Working Group (Italian Longitudinal Study on Ageing). Anthropometric measurements in the elderly: Age and gender differences. Br. J. Nutr. 2002, 87, 177-186. [CrossRef] [PubMed]

36. Jiménez-Jiménez, F.J.; Molina, J.A.; Gómez, P.; Vargas, C.; de Bustos, F.; Benito-León, J.; Tallón-Barranco, A.; Ortí-Pareja, M.; Gasalla, T.; Arenas, J. Neurotransmitter amino acids in cerebrospinal fluid of patients with Alzheimer's disease. J. Neural. Transm. Vienna 1998, 105, 269-277. [CrossRef]

37. Verri, M.; Pastoris, O.; Dossena, M.; Aquilani, R.; Guerriero, F.; Cuzzoni, G.; Venturini, L.; Ricevuti, G.; Bongiorno, A.I. Mitochondrial alterations, oxidative stress and neuroinflammation in Alzheimer's disease. Int. J. Immunopathol. Pharmacol. 2012, 25, 345-353. [CrossRef]

38. Luo, Y.; Yoneda, J.; Ohmori, H.; Sasaki, T.; Shimbo, K.; Eto, S.; Kato, Y.; Miyano, H.; Kobayashi, T.; Sasahira, T.; et al. Cancer usurps skeletal muscle as an energy repository. Cancer Res. 2014, 74, 330-340. [CrossRef]

39. Burns, A.H.; Reddy, W.J. Amino acid stimulation of oxygen and substrate utilization by cardiac myocytes. Am. J. Physiol. 1978, 235, E461-E466. [CrossRef]

40. Barrio, J.R.; Egbert, J.E.; Henze, E.; Schelbert, H.R.; Baumgartner, F.J. L-[4-11C]aspartic acid: Enzymatic synthesis, myocardial uptake, and metabolism. J. Med. Chem. 1982, 25, 93-96. [CrossRef]

41. Grimble, R.F.; Jackson, A.A.; Persaud, C.; Wride, M.J.; Delers, F.; Engler, R. Cysteine and glycine supplementation modulate the metabolic response to tumor necrosis factor alpha in rats fed a low protein diet. J. Nutr. 1992, 122, 2066-2073. [CrossRef] [PubMed]

42. Carubelli, V.; Castrini, A.I.; Lazzarini, V.; Gheorghiade, M.; Metra, M.; Lombardi, C. Amino acids and derivatives, a new treatment of chronic heart failure? Heart Fail. Rev. 2015, 20, 39-51. [CrossRef] [PubMed]

43. Azuma, J.; Sawamura, A.; Awata, N. Usefulness of taurine in chronic congestive heart failure and its prospective application. Jpn. Circ. J. 1992, 56, 95-99. [CrossRef] [PubMed]

44. Kadowaki, M.; Kanazawa, T. Amino acids as regulators of proteolysis. J. Nutr. 2003, 133, 2052S-2056S. [CrossRef] [PubMed] 
45. García-Espinosa, M.A.; Wallin, R.; Hutson, S.M.; Sweatt, A.J. Widespread neuronal expression of branched-chain aminotransferase in the CNS: Implications for leucine/glutamate metabolism and for signaling by amino acids. J. Neurochem. 2007, 100, 1458-1468. [CrossRef]

46. Selkoe, D.J.; Hardy, J. The amyloid hypothesis of Alzheimer's disease at 25 years. EMBO Mol. Med. 2016, 8, 595-608. [CrossRef]

47. Giulietti, A.; Vignini, A.; Nanetti, L.; Mazzanti, L.; Di Primio, R.; Salvolini, E. Alzheimer's disease risk and progression: The role of nutritional supplements and their effect on drug therapy outcome. Curr. Neuropharmacol. 2016, 14, 177-190. [CrossRef]

48. Clements, J.D.; Lester, R.A.; Tong, G.; Jahr, C.E.; Westbrook, G.L. The time course of glutamate in the synaptic cleft. Science 1992, 258, 1498-1501. [CrossRef]

49. Johnson, J.W.; Kotermanski, S.E. Mechanism of action of memantine. Curr. Opin. Pharmacol. 2006, 6, 61-67. [CrossRef]

50. Lipton, S.A. Paradigm shift in neuroprotection by NMDA receptor blockade: Memantine and beyond. Nat. Rev. Drug Discov. 2006, 5, 160-170. [CrossRef]

51. Spencer, S.J.; D'Angelo, H.; Soch, A.; Watkins, L.R.; Maier, S.F.; Barrientos, R.M. High-fat diet and aging interact to produce neuroinflammation and impair hippocampal- and amygdalar-dependent memory. Neurobiol. Aging 2017, 58, 88-101. [CrossRef] [PubMed]

52. Nilsson, L.G.; Nilsson, E. Overweight and cognition. Scand. J. Psychol. 2009, 50, 660-667. [CrossRef] [PubMed]

53. Ardura-Fabregat, A.; Boddeke, E.W.G.M.; Boza-Serrano, A.; Brioschi, S.; Castro-Gomez, S.; Ceyzériat, K.; Dansokho, C.; Dierkes, T.; Gelders, G.; Heneka, M.T.; et al. Targeting neuroinflammation to treat Alzheimer's disease. CNS Drugs 2017, 31, 1057-1082. [CrossRef] [PubMed]

54. Brito, A.; Grapov, D.; Fahrmann, J.; Harvey, D.; Green, R.; Miller, J.W.; Fedosov, S.N.; Shahab-Ferdows, S.; Hampel, D.; Pedersen, T.L.; et al. The human serum metabolome of vitamin B-12 deficiency and repletion, and associations with neurological function in elderly adults. J. Nutr. 2017, 147, 1839-1849. [CrossRef]

55. Wilens, T.E. Effects of methylphenidate on the catecholaminergic system in attention-deficit/hyperactivity disorder. J. Clin. Psychopharmacol. 2008, 28, S46-S53. [CrossRef] [PubMed]

56. Lu, C.D.; Ma, J.K.; Luo, Z.Y.; Tai, Q.X.; Wang, P.; Guan, P.P. Transferrin is responsible for mediating the effects of iron ions on the regulation of anterior pharynx-defective- $1 \alpha / \beta$ and presenilin 1 expression via PGE 2 and $\mathrm{PGD}_{2}$ at the early stage of Alzheimer's disease. Aging Albany N. Y. 2018, 10, 3117-3135. [CrossRef]

57. Oldendorf, W.H. Brain uptake of radiolabeled amino acids, amines, and hexoses after arterial injection. Am. J. Physiol. 1971, 221, 1629-1639. [CrossRef]

58. Chaplin, E.R.; Goldberg, A.L.; Diamond, I. Leucine oxidation in brain slices and nerve endings. J. Neurochem. 1976, 26, 701-707. [CrossRef]

59. Sweatt, A.J.; Wood, M.; Suryawan, A.; Wallin, R.; Willingham, M.C.; Hutson, S.M. Branched-chain amino acid catabolism: Unique segregation of pathway enzymes in organ systems and peripheral nerves. Am. J. Physiol. Endocrinol. Metab. 2004, 286, E64-E76. [CrossRef]

60. Hutson, S.M.; Wallin, R.; Hall, T.R. Identification of mitochondrial branched chain aminotransferase and its isoforms in rat tissues. J. Biol. Chem. 1992, 267, 15681-15686.

61. Hall, T.R.; Wallin, R.; Reinhart, G.D.; Hutson, S.M. Branched chain aminotransferase isoenzymes. purification and characterization of the rat brain isoenzyme. J. Biol. Chem. 1993, 268, 3092-3098. [PubMed]

62. Cooper, A.J.; Plum, F. Biochemistry and physiology of brain ammonia. Physiol. Rev. 1987, 67, 440-519. [CrossRef] [PubMed]

63. Lieth, E.; LaNoue, K.F.; Berkich, D.A.; Xu, B.; Ratz, M.; Taylor, C.; Hutson, S.M. Nitrogen shuttling between neurons and glial cells during glutamate synthesis. J. Neurochem. 2001, 76, 1712-1723. [CrossRef] [PubMed]

64. Hull, J.; Patel, V.; El Hindy, M.; Lee, C.; Odeleye, E.; Hezwani, M.; Love, S.; Kehoe, P.; Chalmers, K.; Conway, M. Regional increase in the expression of the BCAT proteins in Alzheimer's disease brain: Implications in glutamate toxicity. J. Alzheimers Dis. 2015, 45, 891-905. [CrossRef] [PubMed]

65. Gijsman, H.J.; Scarnà, A.; Harmer, C.J.; McTavish, S.B.; Odontiadis, J.; Cowen, P.J.; Goodwin, G.M. A dosefinding study on the effects of branch chain amino acids on surrogate markers of brain dopamine function. Psychopharmacol. Berl. 2002, 160, 192-197. [CrossRef] [PubMed]

66. Hassmén, P.; Blomstrand, E.; Ekblom, B.; Newsholme, E.A. Branched-chain amino acid supplementation during 30-km competitive run: Mood and cognitive performance. Nutrition 1994, 10, 405-410. 
67. Blomstrand, E.; Hassmén, P.; Ek, S.; Ekblom, B.; Newsholme, E.A. Influence of ingesting a solution of branched-chain amino acids on perceived exertion during exercise. Acta Physiol. Scand. 1997, 159, 41-49. [CrossRef]

68. Mittleman, K.D.; Ricci, M.R.; Bailey, S.P. Branched-chain amino acids prolong exercise during heat stress in men and women. Med. Sci. Sports Exerc. 1998, 30, 83-91. [CrossRef]

69. Cerra, F.B.; Cheung, N.K.; Fischer, J.E.; Kaplowitz, N.; Schiff, E.R.; Dienstag, J.L.; Bower, R.H.; Mabry, C.D.; Leevy, C.M.; Kiernan, T. Disease-specific amino acid infusion (F080) in hepatic encephalopathy: A prospective, randomized, double-blind, controlled trial. JPEN J. Parenter. Enter. Nutr. 1985, 9, 288-295. [CrossRef]

70. Jordan, M.K.; Brunner, R.L.; Hunt, M.M.; Berry, H.K. Preliminary support for the oral administration of valine, isoleucine and leucine for phenylketonuria. Dev. Med. Child. Neurol. 1985, 27, 33-39. [CrossRef]

71. Aquilani, R.; Iadarola, P.; Contardi, A.; Boselli, M.; Verri, M.; Pastoris, O.; Boschi, F.; Arcidiaco, P.; Viglio, S. Branched-chain amino acids enhance the cognitive recovery of patients with severe traumatic brain injury. Arch. Phys. Med. Rehabil. 2005, 86, 1729-1735. [CrossRef] [PubMed]

72. Aquilani, R.; Boselli, M.; Boschi, F.; Viglio, S.; Iadarola, P.; Dossena, M.; Pastoris, O.; Verri, M. Branched-chain amino acids may improve recovery from a vegetative or minimally conscious state in patients with traumatic brain injury: A pilot study. Arch. Phys. Med. Rehabil. 2008, 89, 1642-1647. [CrossRef] [PubMed]

73. Cole, J.T.; Mitala, C.M.; Kundu, S.; Verma, A.; Elkind, J.A.; Nissim, I.; Cohen, A.S. Dietary branched chain amino acids ameliorate injury-induced cognitive impairment. Proc. Natl. Acad. Sci. USA 2010, 107, 366-371. [CrossRef] [PubMed]

74. Jeter, C.B.; Hergenroeder, G.W.; Ward 3rd, N.H.; Moore, A.N.; Dash, P.K. Human mild traumatic brain injury decreases circulating branched-chain amino acids and their metabolite levels. J. Neurotrauma 2013, 30, 671-679. [CrossRef] [PubMed]

75. Elkind, J.A.; Lim, M.M.; Johnson, B.N.; Palmer, C.P.; Putnam, B.J.; Kirschen, M.P.; Cohen, A.S. Efficacy, dosage, and duration of action of branched chain amino acid therapy for traumatic brain injury. Front. Neurol. 2015, 6, 73. [CrossRef] [PubMed]

76. Ousset, P.J.; Nourhashemi, F.; Reynish, E.; Vellas, B. Nutritional status is associated with disease progression in very mild Alzheimer disease. Alzheimer Dis. Assoc. Disord. 2008, 22, 66-71. [CrossRef] [PubMed]

77. Chen, Z.; Zhong, C. Decoding Alzheimer's disease from perturbed cerebral glucose metabolism: Implications for diagnostic and therapeutic strategies. Prog. Neurobiol. 2013, 108, 21-43. [CrossRef]

78. Madeira, C.; Lourenco, M.V.; Vargas-Lopes, C.; Suemoto, C.K.; Brandão, C.O.; Reis, T.; Leite, R.E.; Laks, J.; Jacob-Filho, W.; Pasqualucci, C.A.; et al. d-serine levels in Alzheimer's disease: Implications for novel biomarker development. Transl. Psychiatr. 2015, 5, e561. [CrossRef]

79. Bornstein, R.A.; Baker, G.B.; Carroll, A.; King, G.; Wong, J.T.; Douglass, A.B. Plasma amino acids in attention deficit disorder. Psychiatr. Res. 1990, 33, 301-306. [CrossRef]

80. Madras, B.K.; Miller, G.M.; Fischman, A.J. The dopamine transporter and attention-deficit/hyperactivity disorder. Biol. Psychiatr. 2005, 57, 1397-1409. [CrossRef]

(C) 2020 by the authors. Licensee MDPI, Basel, Switzerland. This article is an open access article distributed under the terms and conditions of the Creative Commons Attribution (CC BY) license (http://creativecommons.org/licenses/by/4.0/). 